\title{
Mutation causing congenital myasthenia reveals acetylcholine receptor $\beta / \delta$ subunit interaction essential for assembly
}

\author{
Polly A. Quiram, ${ }^{1}$ Kinji Ohno, ${ }^{2}$ Margherita Milone, ${ }^{2}$ Marc C. Patterson, ${ }^{3}$ Ned J. Pruitt, ${ }^{2}$ \\ Joan M. Brengman, ${ }^{2}$ Steven M. Sine, ${ }^{1}$ and Andrew G. Engel ${ }^{2}$ \\ ${ }^{1}$ Receptor Biology Laboratory, Department of Physiology and Biophysics, \\ ${ }^{2}$ Muscle Research Laboratory, and \\ ${ }^{3}$ Section of Pediatric Neurology, Department of Neurology, Mayo Clinic, Rochester, Minnesota 55905, USA
}

Address correspondence to: Andrew G. Engel, Department of Neurology, Mayo Clinic, Rochester, Minnesota 55905, USA. Phone: (507) 284-5102; Fax: (507) 284-5831; E-mail: age@mayo.edu.

Received for publication August 16, 1999, and accepted in revised form October 5, 1999.

\begin{abstract}
We describe a severe postsynaptic congenital myasthenic syndrome with marked endplate acetylcholine receptor (AChR) deficiency caused by 2 heteroallelic mutations in the $\beta$ subunit gene. One mutation causes skipping of exon 8,truncating the $\beta$ subunit before its M1 transmembrane domain, and abolishing surface expression of pentameric AChR. The other mutation, a 3-codon deletion ( $\beta 426 \mathrm{delEQE})$ in the long cytoplasmic loop between the M3 and M4 domains, curtails but does not abolish expression. By coexpressing $\beta 426 \mathrm{delEQE}$ with combinations of wild-type subunits in $293 \mathrm{HEK}$ cells, we demonstrate that $\beta 426$ delEQE impairs AChR assembly by disrupting a specific interaction between $\beta$ and $\delta$ subunits. Studies with related deletion and missense mutants indicate that secondary structure in this region of the $\beta$ subunit is crucial for interaction with the $\delta$ subunit. The findings imply that the mutated residues are positioned at the interface between $\beta$ and $\delta$ subunits and demonstrate contribution of this local region of the long cytoplasmic loop to AChR assembly.
\end{abstract}

J. Clin. Invest. 104:1403-1410 (1999).

\section{Introduction}

The protein sequence of ion channels governs not only their ultimate function, but also encodes instructions for their correct assembly. Converting the linear peptide into the mature protein requires correct folding, posttranslational modification, and, for most ion channels, oligomerization (1). For the acetylcholine receptor $(\mathrm{AChR})$ at the motor endplate (EP), these steps likely depend on local sequences in many parts of its $\alpha, \beta, \varepsilon$, and $\delta$ subunits. Identifying such key assembly sequences typically relies on mutating residues conserved across the AChR superfamily. However, by identifying the genetic defects underlying a congenital myasthenic syndrome (CMS), the present work reveals a region of the AChR $\beta$ subunit essential for assembly.

The amino-terminal, extracellular half of each AChR subunit is widely recognized to mediate its initial association leading to the assembled pentamer $(2,3)$. A cystine loop within the extracellular domain, formed between C128 and C142 in all AChR subunits, has drawn considerable attention regarding its role in contributing to assembly. Formation of cystine loops in both $\alpha$ and $\beta$ subunits is required for specific conformational changes and subunit oligomerization steps at intermediate stages of assembly (4). Furthermore, specific residues preceding the cystine loop affect assembly efficiency (5), whereas residues following the loop govern subunit specificity of oligomerization (6). On the other hand, residues in the M1 and M2 transmem- brane domains are essential for assembly of homomeric versus heteromeric AChRs (7).

We now uncover an additional domain essential for AChR assembly by identifying and characterizing the molecular defects that cause a severe CMS associated with marked EP-AChR deficiency. The deficiency arises from 2 heteroallelic recessive mutations in the $\beta$ subunit. One causes skipping of exon 8 , which abolishes expression of pentameric AChR; the second is a 3-codon deletion ( $\beta 426 \mathrm{delEQE})$ in the long cytoplasmic loop between transmembrane domains M3 and M4, which severely curtails expression of cell-surface AChR. By coexpressing $\beta 426$ delEQE and related deletion mutants with combinations of wild-type subunits, we demonstrate that $\beta 426$ delEQE impairs AChR assembly by disrupting a specific interaction between $\beta$ and $\delta$ subunits.

\section{Methods}

Muscle specimens. Intercostal muscle specimens were obtained intact from origin to insertion from the patient and control subjects without muscle disease undergoing thoracic surgery. A limb-muscle specimen was obtained from the patient's mother. All human studies were in accord with the guidelines of the Institutional Review Board of the Mayo Clinic.

AChR and acetylcholinesterase (AChE) were localized in cryostat sections by 2-color fluorescence (8). EPs were localized for electron microscopy (9) and quantitatively analyzed (10) by established methods. Peroxidase-labeled $\alpha$-bun- 

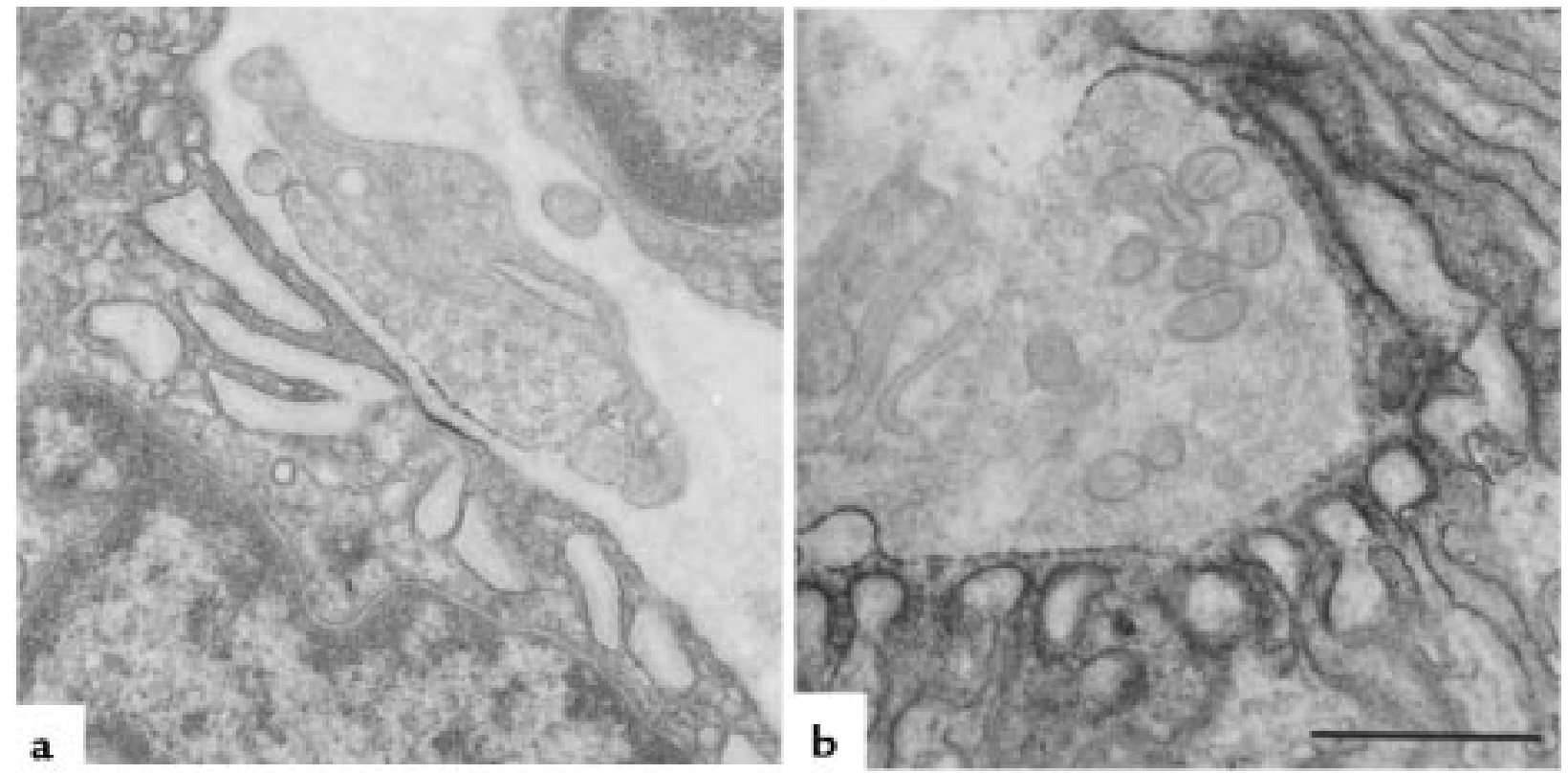

Figure 1

EP fine structure and localization of AChR at patient (a) and control subject (b) EP regions with peroxidase-labeled $\alpha$-bgt. Note the small nerve terminal, small postsynaptic region, no openings from the primary synaptic cleft into secondary clefts, and patchy and attenuated reaction for AChR in patient EP.

garotoxin ( $\alpha$-bgt) was used for the ultrastructural localization of AChR (11). The number of AChRs per EP was measured with $\alpha$-bgt labeled with ${ }^{125}$ I, as described (12).

Electrophysiology of muscle specimens. Miniature EP (MEPP), miniature EP current (MEPC), and EP potential recordings, estimates of the number of transmitter quanta released by nerve impulse, and analysis of the ACh-induced current noise were carried out as described previously $(12,13)$. Patch-clamp recordings from the EP were performed in the cell-attached mode by a method described previously (14).

Mutation analysis. We directly sequenced the $\mathrm{AChR} \alpha, \beta$, $\delta$, and $\varepsilon$ subunit genes using genomic DNA and mRNA as described elsewhere (15). We searched for the cause of the skipping of $\beta$ exon 8 by examining all putative cis-acting elements of introns 7 and 8, comprising the first 222 and the last 161 nucleotides of intron 7 and the entire 757 nucleotides of intron 8; by long-distance PCR and

\section{Table 1}

Endplate studies

\begin{tabular}{|c|c|c|}
\hline & Patient & Control subjects \\
\hline$\left[{ }^{125} \mid\right] \alpha$-bgt binding sites/EP & \multicolumn{2}{|c|}{$\begin{array}{r}0.31 \mathrm{E} 6 \mathrm{12.82} \pm 0.79 \mathrm{E} 6 \text { ( } 13 \text { adults) } \\
4.7 \mathrm{E} 6 \text { ( } 3 \text {-year-old control subject) }\end{array}$} \\
\hline EPP quantal content $(1 \mathrm{~Hz})^{\mathrm{A}}$ & $39 \pm 3(15)$ & $31 \pm 1(190)$ \\
\hline MEPP amplitude $(\mathrm{mV})^{\mathrm{B}}$ & $0.18 \pm 0.011(16)$ & $1.00 \pm 0.025(164)$ \\
\hline MEPC amplitude $(n A)^{C}$ & $1.08 \pm 0.05$ & $3.95 \pm 0.10(79)$ \\
\hline$\tau_{\text {MEPC }}(\mathrm{ms})^{\mathrm{C}}$ & $2.77 \pm 0.18(12)$ & $3.23 \pm 0.06(79)$ \\
\hline$\tau_{\text {noise }}(\mathrm{ms})^{\mathrm{C}}$ & $2.14 \pm 0.18$ & $2.3 \pm 0.043(52)$ \\
\hline
\end{tabular}

Values represent mean $\pm \mathrm{SE}$; numbers in parentheses indicate number of EPs, except for $\alpha$-bgt-binding studies, where they indicate number of adult control subjects. T $=29 \pm 0.5^{\circ} \mathrm{C}$ for EPP and MEPP recordings, and $22 \pm 0.5^{\circ} \mathrm{C}$ for noise analysis and MEPC studies. ${ }^{\mathrm{A}} \mathrm{Quantal}$ content of EP potential (EPP) at $1-\mathrm{Hz}$ stimulation, corrected for resting membrane potential of $-80 \mathrm{mV}$, nonlinear summation, and nonPoisson release. ${ }^{B}$ Corrected for resting membrane potential of $-80 \mathrm{mV}$ and a mean muscle fiber diameter of $55 \mu \mathrm{m}$. ${ }^{\mathrm{C}}-80 \mathrm{mV}$. restriction analysis to detect a large-scale DNA deletion involving exon 8; and by long-distance PCR to identify Alu-mediated inversion and duplication of exon 8.

To trace $\beta 1276$ del9 in family members, we size-fractionated PCR products. To trace $\beta 1276 \mathrm{del} 9$ in 100 normal control subjects, we employed allele-specific PCR. To prove that all affected siblings carried the same mutant alleles as the propositus, we analyzed 5 single nucleotide polymorphisms ( $\varepsilon 1233 \mathrm{C} / \mathrm{T}, \beta \mathrm{E} 9 \mathrm{G}$, $\beta \mathrm{IVS} 6+6 \mathrm{~T} / \mathrm{C}$, $\beta$ IVS6$62 \mathrm{~A} / \mathrm{G}$, and $\beta \mathrm{IVS} 10-145 \mathrm{~A} / \mathrm{G})$ in the AChR $\varepsilon$ and $\beta$ subunit genes and 3 microsatellite markers (D17S969, D17S918, and D17S2196) in family members. PCR primers, PCR conditions, and restriction enzymes, if applicable, are available upon request.

Construction and expression of wild-type and mutant AChRs. Human AChR $\alpha, \beta, \delta$, and $\varepsilon$ subunit cDNA were cloned into the CMV-based expression vector pRBG4, as described elsewhere (16). The $\beta 1276$ del9 mutation, skipping of $\beta$ exon 8 , and the other artificial mutations were introduced into the $\beta$ subunit cDNA in pRBG4 by the QuickChange Site-Directed Mutagenesis kit (Stratagene, La Jolla, California, USA) or by its megaprimer-based modification (17). Human embryonic kidney (HEK) 293 cells were transfected with AChR subunit cDNAs using calcium phosphate precipitation (16).

Total $\alpha$-bgt binding measurements. The total number of $\left[{ }^{125} \mathrm{I}\right] \alpha$-bgt sites was determined in triplicate, as described previously (16).

Sucrose gradient centrifugation. HEK cells expressing AChR oligomers were permeabilized with $1 \%$ saponin, labeled with [125I] $\alpha$-bgt, washed free of unbound radioactivity, and solubilized in Triton X-100 buffer (0.6\% Triton $\mathrm{X}-100,150 \mathrm{mM}$ sodium chloride $[\mathrm{NaCl}], 5 \mathrm{mM}$ EDTA, $50 \mathrm{mM}$ Tris, $35 \mu \mathrm{g} / \mathrm{mL}$ PMSF, $10 \mu \mathrm{g} / \mathrm{mL}$ aprotinin, and $1 \mu \mathrm{g} / \mathrm{mL}$ pepstatin $\mathrm{A}, \mathrm{pH}$ 7.5). Extracts were 
layered on sucrose gradients (3-30\%), centrifuged for 22 hours at 40,000 rpm in an SW-41 rotor, and fractions were collected and counted with a gamma counter.

Patch-clamp recordings from AChRs expressed in HEK cells. Recordings were obtained in the cell-attached configuration with an applied membrane potential of $-70 \mathrm{mV}$, a temperature of $22^{\circ} \mathrm{C}$, and with bath and pipette solutions containing $(\mathrm{mM})$ : potassium chloride $(\mathrm{KCl}) 142$, $\mathrm{NaCl} 5.4$, calcium chloride $\left(\mathrm{CaCl}_{2}\right) 1.8$, magnesium chloride $\left(\mathrm{MgCl}_{2}\right)$ 1.7, HEPES 10, pH 7.4 (18). Single-channel currents were acquired and processed for analysis as described previously (16). Open- and closed-duration histograms were constructed using a logarithmic abscissa and square root ordinate (19), and fitted to the sum of exponentials by maximum likelihood.

\section{Results}

Characteristics of CMS patient. A female, now 8 years of age, had severe myasthenic symptoms since birth, requiring frequent ventilation and enteric alimentation through a gastrostomy. She had a decremental electromyographic response on stimulation of motor nerves and responded partially to cholinesterase inhibitors. Tests for anti-AChR antibodies were negative. Two younger siblings are similarly affected; the parents are unaffected. An intercostal muscle was obtained from the patient at 4 years of age.

Endplate studies. The configuration of the EPs evaluated from the cytochemical reaction for AChE on longitudinally oriented, teased, single muscle fibers was abnormal, with an increased number of small EP regions distributed over a 3-fold increased span of the muscle fiber surface. Severe EP-AChR deficiency was established by several measures. The number of [ $\left.{ }^{125} \mathrm{I}\right] \alpha$-bgt sites per EP was less than $3 \%$ of the normal adult value and less than $7 \%$ of the value obtained in a 3 -year-old control (Table 1). The reaction for AChR at the EPs, detected in cryostat sections with rhodamine-labeled $\alpha$-bgt, was markedly attenuated. Quantitative electron microscopy studies of 37 EP regions at 17 EPs revealed that the structural integrity of the pre- and postsynaptic regions was preserved, but nerve terminal size was decreased to $62 \%$ of normal and the postsynaptic area of folds and clefts was only $29 \%$ of normal. The number of secondary synaptic clefts per micrometer length of the primary cleft was reduced to $53 \%$ of normal. The reaction for AChR, detected with peroxidase-labeled $\alpha$-bgt, was patchy and attenuated (Figure 1, a and b). The AChR index (defined as the ratio of the postsynaptic membrane reacting for AChR to the length of the primary synaptic cleft) was only $11 \%$ of the control mean.

Quantal release by nerve impulse was normal. The amplitude of the MEPPs was reduced to $18 \%$ and that of the MEPCs to about $25 \%$ of normal. The MEPC decay time constant was normal, indicating that the duration of most or all AChR channel-opening episodes was normal. Spectral analysis of the ACh-induced current noise gave similar results (Table 1). The frequency of channel events during single-channel patch-clamp recordings with 0.1 to $10 \mu \mathrm{M}$ ACh was very low, so that only 600 bursts of openings could be recorded from 5 EPs. Analysis of the pooled recordings revealed that $89 \%$ of the bursts had a normal conductance of approximately $61 \mathrm{pS}$, and the dwell time of the dominant burst component, $3.27 \mathrm{~ms}$, was similar to the $3.04 \pm 0.18 \mathrm{~ms}$ found at 34 control EPs (20). Eleven percent of the bursts had a conductance of approximately $46 \mathrm{pS}$ and a 2 -fold longer than normal open duration, typical of fetal receptors

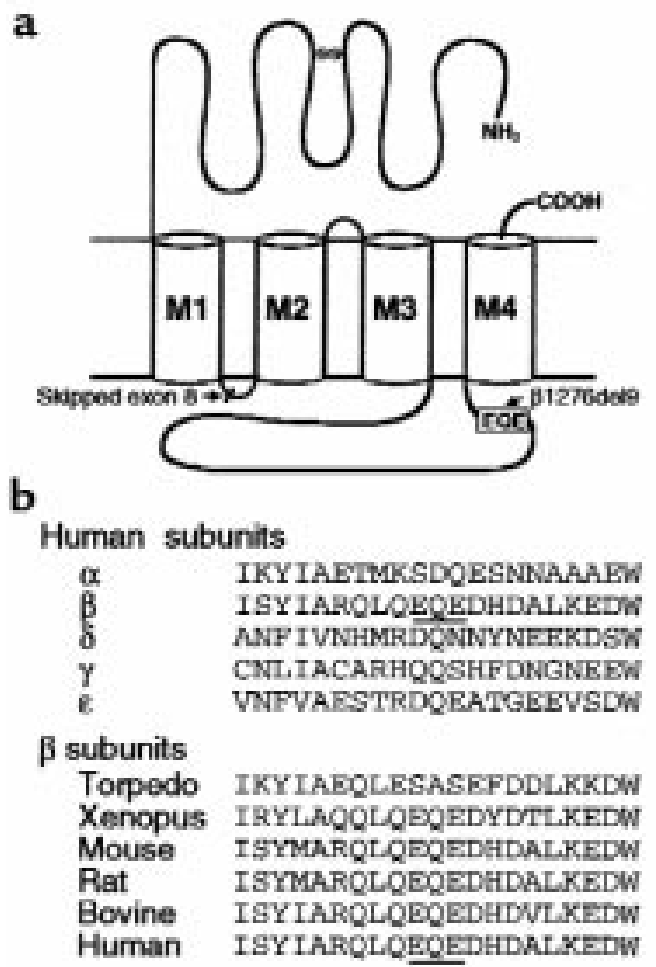

C

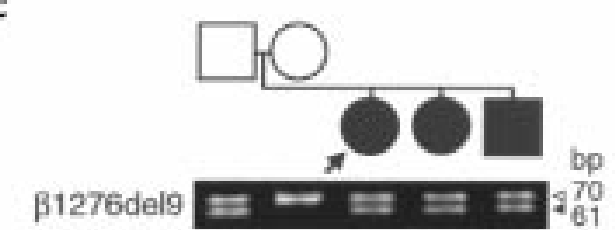

d

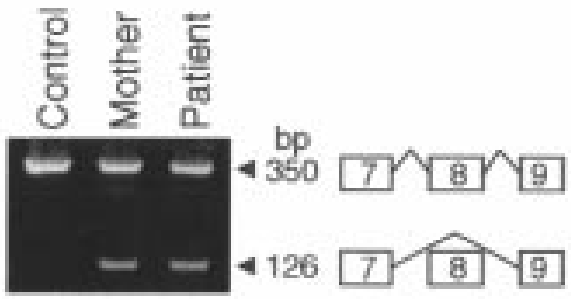

Figure 2

(a) Schematic presentation of the positions of the identified mutations in the AChR $\beta$ subunit gene. (b) Multiple alignment of the long cytoplasmic loop of AChR subunits around the $\beta 1276$ del9 mutation. $\beta 1276$ del9 predicts an in-frame deletion of EQE codons at $426-428$ (underlined). (c) Size fractionation of $P$ stl-digested PCR products amplified from genomic DNA from blood of family members. The $\beta 1276 \mathrm{del} 9$ mutation results in a 61-bp fragment, whereas the wild-type allele gives rise to a 70-bp fragment. The father and 3 affected siblings are heterozygous for $\beta 1276$ del9. Arrow indicates patient; filled symbols indicate affected individuals. (d) Size fractionation of RT-PCR products of the AChR $\beta$ subunit gene amplified with primers in exon 7 and 9. The normal transcript yields a 350-bp fragment; skipping of exon 8 results in a 126bp fragment. Mother and patient carry both transcripts. Skipping of exon 8 was confirmed by direct sequencing. 
that harbor the $\gamma$ instead of the $\varepsilon$ subunit (21). To summarize, EP studies revealed severe AChR deficiency, no kinetic abnormality of the prevailing population of AChRs, and presence of a small population of fetal AChRs.

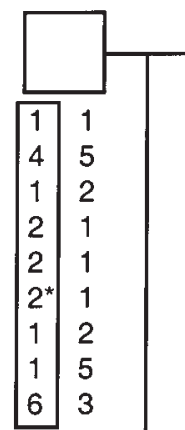

$12 \varepsilon 1233 \mathrm{C} / \mathrm{T}$ : $12.2 \mathrm{cM}$ pterm

3 4 D17S969: $6.0 \mathrm{cM}$ pterm

12 BE9G

1 (1) $\mathrm{BIVS} 6+6 \mathrm{~T} / \mathrm{C}$

1 1 3 IVS6-62AVG

11 B1276del9

2 2 BIVS10-145A/G

15 D17S918: $2.5 \mathrm{cM}$ qterm

41 D17S2196: $5.3 \mathrm{cM}$ qterm

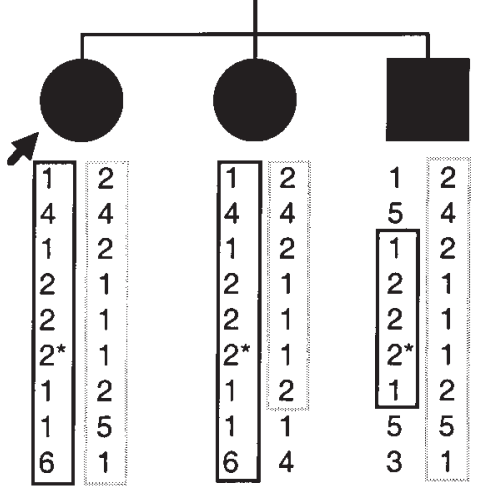

Figure 3

Haplotype analysis of family members using $\beta 1276$ del9, 5 single nucleotide polymorphisms, and 3 microsatellite markers. The 3 affected siblings carry the same haplotypes for the $\beta$ subunit gene. The allele with $\beta 1276 \mathrm{del} 9$ (asterisk) is shown by a solid box; the allele expected to cause skipping of $\beta$ exon 8 is shown by a dotted box. Distances from the $\beta$ subunit gene locus are shown in centimorgans (cM). pterm, p-terminal direction; qterm, q-terminal direction.
Mutation analysis. Direct sequencing of all exons and flanking regions of $\mathrm{AChR} \alpha, \beta, \delta$, and $\varepsilon$ subunit genes revealed a heterozygous 9-bp deletion at nucleotides $1276-1284$ in $\beta$ exon 10 ( $\beta 1276$ del9), predicting an inframe deletion of Glu-Gln-Glu codons at 426-428 ( $\beta 426$ delEQE) in the long cytoplasmic loop (Figure 2, a and b). $\beta 1276 \mathrm{del} 9$ was not found in 100 normal controls.

To search for a second mutation, we directly sequenced overlapping cDNA fragments of the $\beta$ subunit gene. This revealed heterozygous skipping of exon 8. Skipping of exon 8 (224 bp) causes a frameshift after codon 250 in the short cytoplasmic link between the M1 and M2 domains, predicting 40 missense codons followed by a stop codon (Figure 2a). We next wished to determine the cause of exon skipping. Sequencing of wild-type introns 7 and 8 revealed 11 and 1 Alu repeats, respectively (data not shown). Therefore, we first screened for Alu-mediated deletion, duplication, and inversion around exon 8 , but detected none. We next sequenced all putative cis-acting elements of intron 7 that affect splicing, as well as entire intron 8 , but found no mutations or polymorphisms.

Analysis of family members showed that for each affected sibling, the father transmitted $\beta 1276$ del9 (Figures $2 \mathrm{c}$ and 3 ), the mother transmitted a mutation causing skipping of $\beta$ exon 8 (Figures $2 \mathrm{~d}$ and 3 ), and the mutations are heteroallelic and recessive.

AChR expression in HEK cells. To evaluate pathogenicity of the mutant $\beta$ subunits, we cotransfected them with complementary $\alpha, \delta$, and $\varepsilon$ subunits in 293 HEK cells and measured binding of [125I] $\alpha$-bgt to cell-surface receptors. Expression in the presence of the $\beta$ subunit with skipped exon 8 reduces expression to $3.3 \%$ of control. On the other hand, coexpressing the $\beta 1276 \mathrm{del} 9$ ( $\beta 426 \mathrm{delE}-$ QE) subunit with complementary wild-type subunits reduces expression to approximately $30 \%$ of control, indicating low but detectable expression of AChRs containing $\beta 426$ delEQE (Figure 4a). a

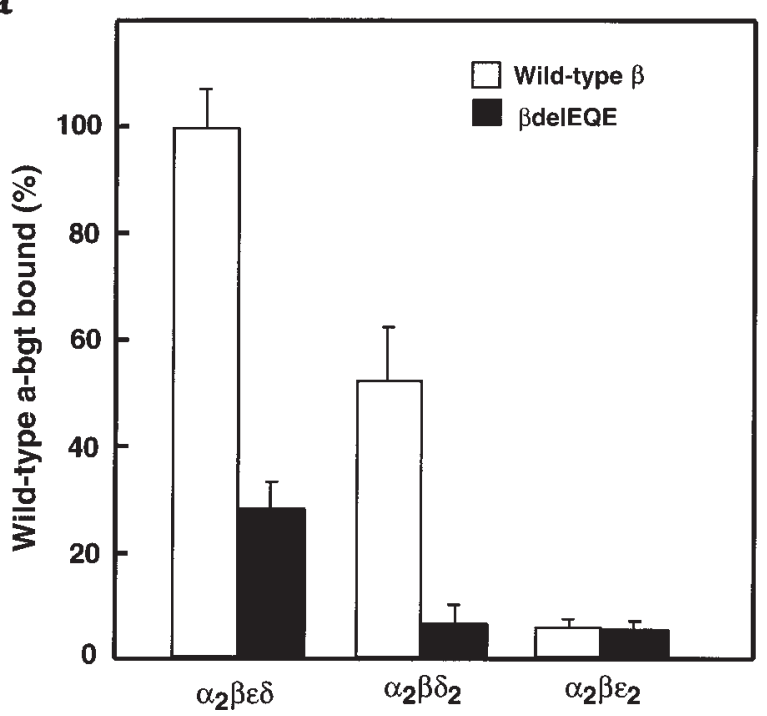

b

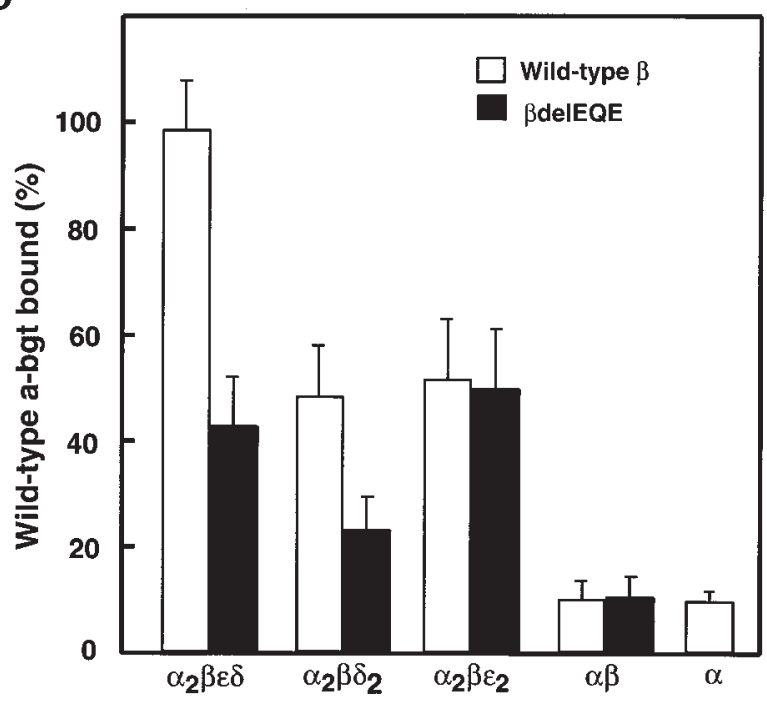

Figure 4

Expression of pentameric AChRs containing wild-type or mutant $\beta$ subunits. (a) $\alpha$-bgt binding to surface receptors on intact HEK cells transfected with the indicated AChR subunits. (b) Total $\alpha$-bgt binding to saponin-permeabilized cells transfected with the indicated AChR subunits. Amounts of bound [ $\left.{ }^{125} I\right] \alpha$-bgt are normalized to that measured for wild-type $\operatorname{AChR}\left(\alpha_{2} \beta \varepsilon \delta\right)$. 
To determine whether $\beta 426$ delEQE reduces expression through an impaired interaction with another subunit, we measured expression of pentamers lacking either an $\varepsilon$ or $\delta$ subunit but containing either wild-type or mutant $\beta$ subunits. Omission of either $\varepsilon$ or $\delta$ subunits leads to cell-surface pentamers (22), $\alpha_{2} \beta \delta_{2}$ or $\alpha_{2} \beta \varepsilon_{2}$, allowing studies of subunit-subunit interactions. The mutant $\beta 426$ delEQE markedly reduces expression of $\alpha_{2} \beta \delta_{2}$ pentamers, but does not affect expression of $\alpha_{2} \beta \varepsilon_{2}$ pentamers (Figure 4a). Thus, the reduced expression is specific to pentamers containing the $\delta$ subunit, suggesting an impaired interaction between $\beta$ and $\delta$ subunits in the course of receptor assembly.

To determine whether the $\beta 426$ delEQE subunit reduces the total cellular pool of receptors, we measured $\left[{ }^{125} \mathrm{I}\right] \alpha$-bgt binding to saponin-permeabilized cells expressing various combinations of subunits (Figure $4 b$ ). As observed for cell-surface receptors, the $\beta 426 \mathrm{delEQE}$ subunit reduces the total cellular pool of pentamers containing the $\delta$ subunit, $\alpha_{2} \beta \varepsilon \delta$ and $\alpha_{2} \beta \delta_{2}$, but does not affect the total pool of $\alpha_{2} \beta \varepsilon_{2}$ pentamers. Additionally, coexpression of $\alpha$ and $\beta 426 \mathrm{delEQE}$ subunits produces normal amounts of $\alpha \beta$ complexes, indicating that the interaction between $\alpha$ and $\beta$ subunits is maintained. Thus, the $\beta 426$ delEQE subunit reduces the total cellular pool as long as the receptors contain the $\delta$ subunit.

The $\beta 426$ delEQE mutation could reduce expression by either disrupting a secondary structure, such as an $\alpha$ helix, or by removing key side chains involved in intrasubunit interactions. To distinguish between these possibilities, we made additional deletions and amino acid substitutions in this region of the $\beta$ subunit, coexpressed each with complimentary $\alpha, \delta$, and $\varepsilon$ subunits, and measured $\left.{ }^{125 I}\right] \alpha$-bgt binding to the cell surface. Similar to $\beta 426$ delEQE, deletion of 2,4 , or 6 residues in this region of the $\beta$ subunit also reduces surface expression, with the largest affect observed with the 6-residue deletion (Figure 5a). These deletion experiments suggest that $\beta 426$ delEQE disrupts the secondary structure of the long cytoplasmic loop of the $\beta$ subunit, which is a putative amphipathic $\alpha$ helix (23). Conversely, replacing EQE in the wild-type $\beta$ subunit with the triple mutations AAA or KQK does not affect surface expression, indicating that the amino acid sequence in this region is not decisive for receptor expression. Thus, reduction of surface expression caused by $\beta 426$ delEQE is likely because of an altered secondary structure at the distal end of the long cytoplasmic loop of the $\beta$ subunit.

To determine whether the series of deletion and triple point mutations of the $\beta$ subunit affect the total cellular pool of receptors, we coexpressed them with $\alpha, \delta$, and $\varepsilon$ subunits and measured [125I] $\alpha$-bgt binding following permeabilization with saponin (Figure 5b). The 2-, 4-, and 6 -residue deletion mutations reduce the total cellular pool of receptors, whereas the AAA and KQK mutations are without effect. Interestingly, the 6-residue deletion mutation reduces cell-surface more than total AChR expression, indicating that this region of the $\beta$ subunit contributes not only to subunit oligomerization but also to stability of receptors on the cell surface.

To determine whether our engineered $\beta$ subunit deletions and triple point mutations specifically affect recep- tors containing a $\delta$ subunit, we examined expression of subunit-omitted pentamers, $\alpha_{2} \beta \delta_{2}$ and $\alpha_{2} \beta \varepsilon_{2}$, containing either wild-type or mutant $\beta$ subunits. The 2-, 4-, and 6residue deletion mutations reduce surface expression of $\alpha_{2} \beta \delta_{2}$ pentamers, but do not affect surface expression of $\alpha_{2} \beta \varepsilon_{2}$ pentamers (Figure 6a), again pointing to an impaired interaction between $\beta$ and $\delta$ subunits. The triple mutation AAA does not affect expression of either $\alpha_{2} \beta \delta_{2}$
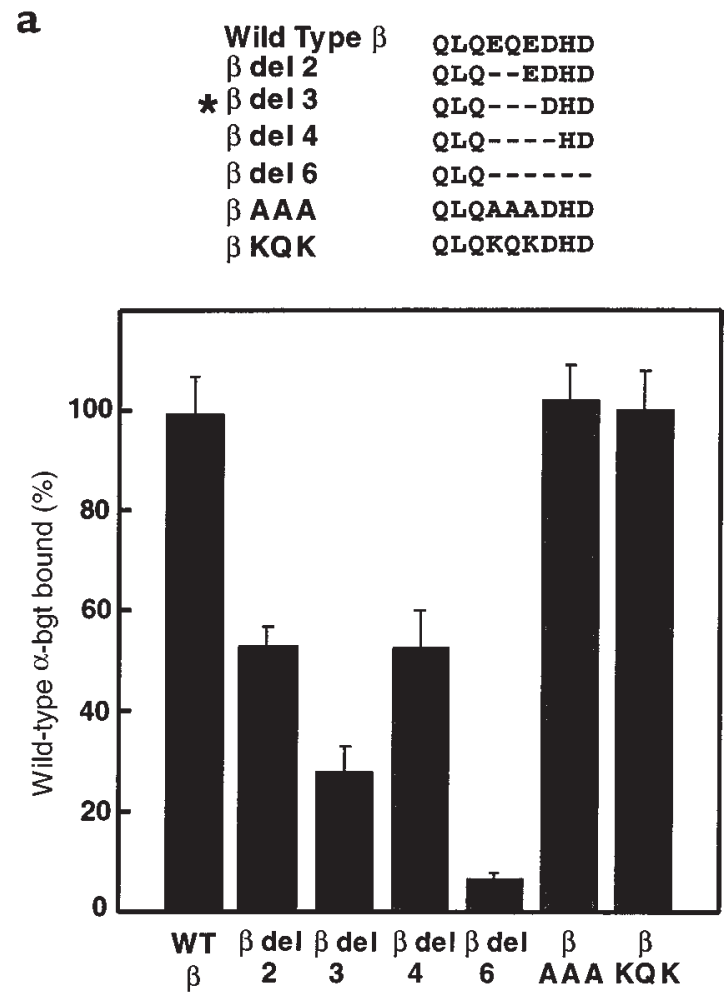

b

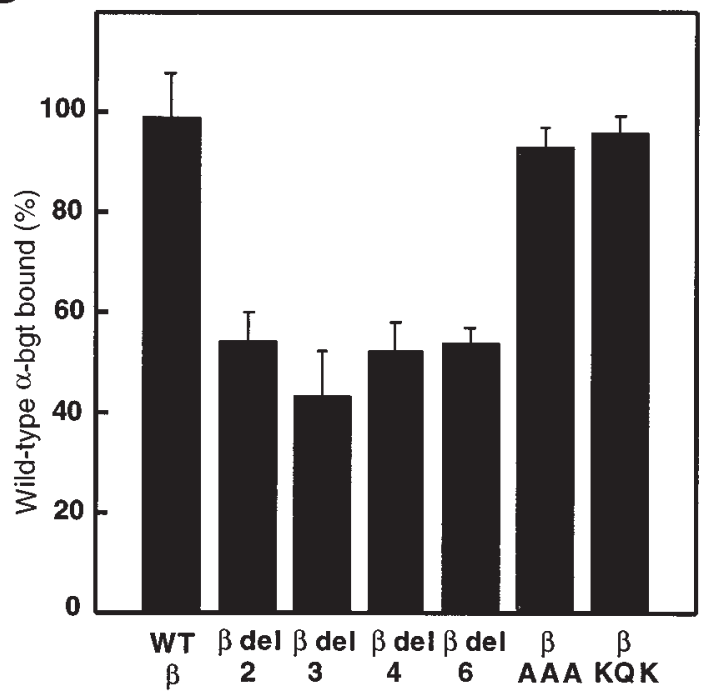

Figure 5

Expression of pentameric AChRs containing mutant $\beta$ subunits. Local sequences of the mutant $\beta$ subunits are shown at the top, with the asterisk indicating the patient mutation. (a) $\alpha$-bgt binding to cell-surface receptors from HEK cells transfected with the indicated $\beta$ subunits along with complimentary $\alpha, \varepsilon$, and $\delta$ subunits. (b) Same determinations as a, but with saponin-permeabilized cells. Amounts of bound $\left[{ }^{125}\right.$ I $] \alpha$-bgt are normalized as in Figure 4. 
or $\alpha_{2} \beta \varepsilon_{2}$ pentamers. However, the mutation KQK, which replaces 2 negatively charged residues with positively charged ones, markedly increases surface expression of

a

$\begin{array}{cc}\text { Wild Type } \beta & \text { QLQEQEDHD } \\ \beta \text { del } 2 & \text { QLQ--EDHD } \\ \text { * } \beta \text { del } 3 & \text { QLQ---DHD } \\ \beta \text { del } 4 & \text { QLQ----HD } \\ \beta \text { del } 6 & \text { QLQ------ } \\ \beta \text { AAA } & \text { QLQAAADHD } \\ \beta \text { KQK } & \text { QLQKQKDHD }\end{array}$
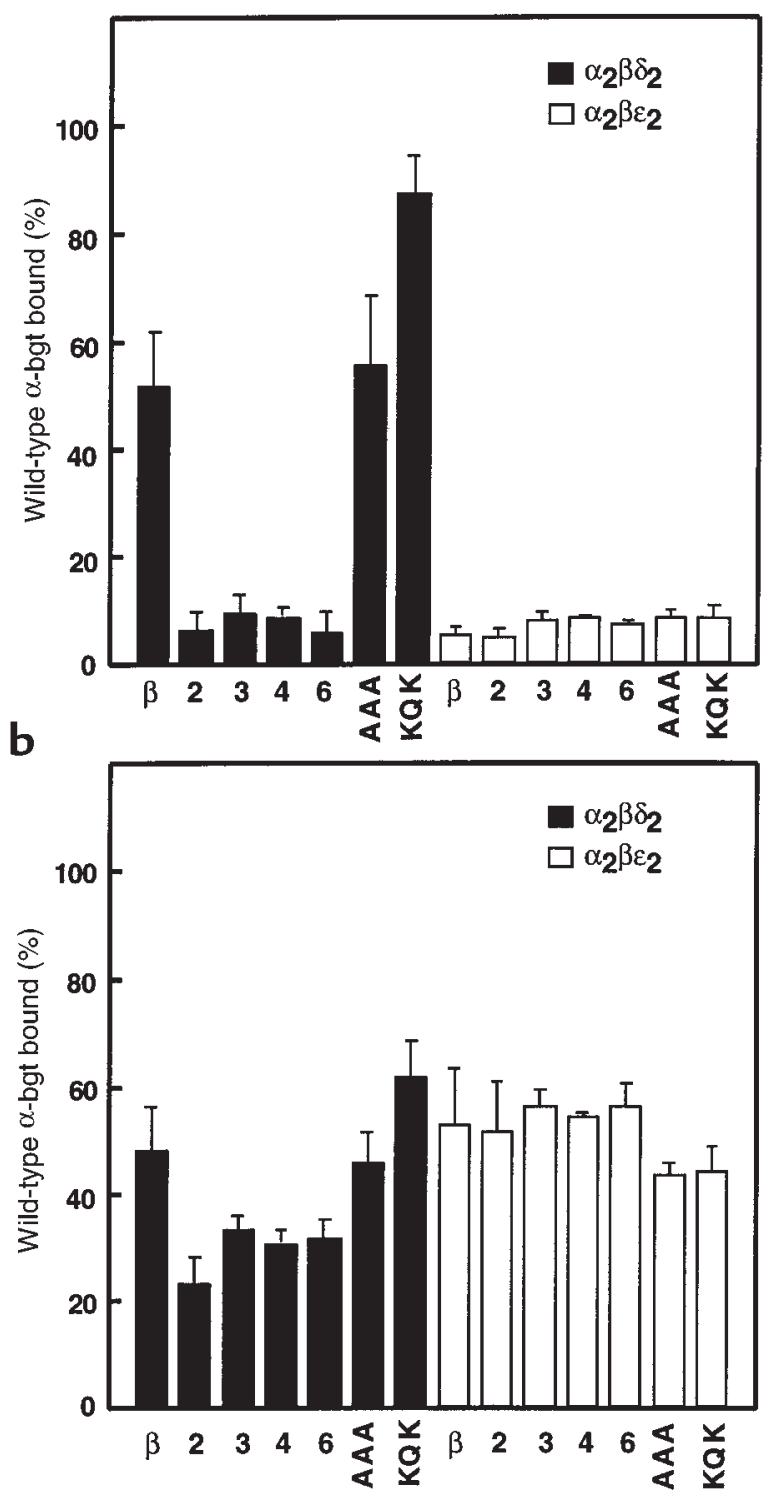

Figure 6

Expression of subunit-omitted $\alpha_{2} \beta \delta_{2}$ and $\alpha_{2} \beta \varepsilon_{2}$ pentamers containing mutant $\beta$ subunits. Local sequences of the mutant $\beta$ subunits are shown at the top, with the asterisk indicating the patient mutation. (a) $\alpha$-bgt binding to surface receptors on intact HEK cells transfected with either $\alpha, \beta$, and $\delta$ subunits (to form $\alpha_{2} \beta \delta_{2}$ ) or $\alpha, \beta$, and $\varepsilon$ subunits (to form $\alpha_{2} \beta \varepsilon_{2}$ ). For each combination of subunits, the type of $\beta$ subunit is indicated on the $x$ axis. (b) Same determinations as a, but with saponin-permeabilized cells. Amounts of bound [ $\left.{ }^{125} \mid\right] \alpha$-bgt are normalized as in Figure 4. $\alpha_{2} \beta \delta_{2}$ pentamers, without affecting expression of $\alpha_{2} \beta \varepsilon_{2}$ pentamers. These mutated $\beta$ subunits preferentially affect pentamers containing a $\delta$ subunit, again suggesting disruption of a specific interaction between $\beta$ and $\delta$ subunits.

To determine whether the series of deletion and triple mutations affect the total cellular pool of $\alpha_{2} \beta \delta_{2}$ and $\alpha_{2} \beta \varepsilon_{2}$ pentamers, we saponin-permeabilized cells expressing subunit-omitted pentamers containing either wild-type or mutant $\beta$ subunits (Figure $6 \mathrm{~b}$ ). Measurements of $\left[{ }^{125} \mathrm{I}\right] \alpha$-bgt binding to the total pool of receptors mirror our observations for cell-surface pentamers: the deletion mutations reduce expression of $\alpha_{2} \beta \delta_{2}$ pentamers without affecting expression of $\alpha_{2} \beta \varepsilon_{2}$ pentamers, whereas the AAA and KQK mutations are without effect. Again, the deletion mutations reduce cell-surface more than total cellular expression, indicating that the mutations affect stability of receptors on the cell surface.

Sucrose gradient sedimentation of receptors containing $\beta 426$ delEQE. To determine whether $\beta 426$ delEQE causes buildup of assembly intermediates, we examined by sucrose gradient sedimentation total cellular extracts from HEK cells expressing either $\alpha, \beta$, $\varepsilon$, and $\delta$, or $\alpha, \beta$, and $\delta$, in which $\beta$ was either wild-type or the $\beta 426 \mathrm{del}$ $\mathrm{QE}$ subunit (Figure 7). The resulting sedimentation profiles show 2 distinct peaks: a $1.3 \mathrm{~S}$ peak corresponding to free $\left[{ }^{125} \mathrm{I}\right] \alpha$-bgt, and a $9.0 \mathrm{~S}$ peak corresponding to $\mathrm{AChR}$ pentamers. No peaks with intermediate sedimentation coefficients were detected, indicating that $\beta 426 \mathrm{delEQE}$ does not cause buildup of AChR assembly intermediates.

Function of AChRs containing $\beta 426$ delEQE. We next examined single channel currents activated by $10 \mu \mathrm{M}$ ACh from HEK cells expressing AChRs containing either wild-type or mutant $\beta$ subunits (Figure 8 ). We found that both wildtype and mutant receptors open and close with similar kinetics. In particular, the open duration histograms are similar for both receptor types, each consisting of a dominant component with a mean duration of $1 \mathrm{~ms}$, as observed previously for human AChR (16). The closedduration histograms contain 2 major components, both of which have similar mean durations and relative areas for wild-type and mutant receptors. Thus, receptor function is not detectably altered by the $\beta 426$ delEQE subunit.

To summarize, the expression studies reveal that $\beta 426$ delEQE reduces expression of surface receptors through an impaired interaction between $\beta$ and $\delta$ subunits. The mutant receptors that do assemble are structurally and functionally similar to receptors containing the wild-type $\beta$ subunit, but their expression efficiency is markedly curtailed.

\section{Discussion}

Congenital myasthenic syndromes. CMS is an inherited disorder in which the safety margin of neuromuscular transmission is impaired by one or more specific mechanisms (24). The CMSs identified to date stem from presynaptic, synaptic, or postsynaptic defects. Molecular genetic studies indicate that the postsynaptic CMSs are caused by mutations in AChR subunits (reviewed in ref. 25).

Dominant gain-of-function mutations in AChR subunits increase the response to ACh by prolonging opening episodes of the receptor. On the other hand, recessive lossof-function mutations reduce affinity for $\mathrm{ACh}$, disturb the 


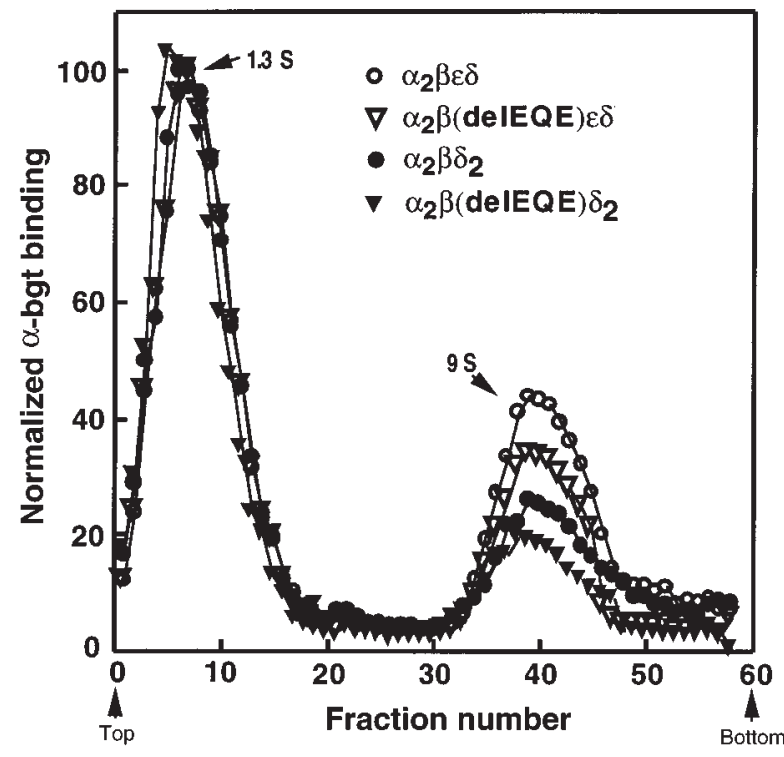

Figure 7

Sucrose gradient centrifugation of AChRs from saponin-permeabilized cells transfected with the indicated subunits. Following saponin treatment, cells were incubated with $\left[{ }^{125} \mid\right] \alpha$-bgt, washed free of unbound $\alpha$ bgt, solubilized, and centrifuged on sucrose gradients as described in Methods. Radioactivity in each fraction is normalized to the peak value in each gradient. Arrows and $\mathrm{S}$ values indicate $\alpha$-bgt (1.3 S) and $\alpha_{2} \beta \delta \varepsilon$ or $\alpha_{2} \beta \varepsilon_{2}$ pentamer ( $9 \mathrm{~S}$ ) peaks.

gating mechanism, or reduce AChR expression. Marked decrease of AChR expression is caused by mutations in AChR subunit genes that prematurely terminate the translational chain $(17,26-28)$, alter the signal peptide region (16), or affect residues essential for assembly of the pentameric receptor $(16,26,29)$. Most of these mutations occur in the $\varepsilon$ subunit gene, because compensatory expression of the fetal-type AChR that harbors the $\gamma$ instead of the $\varepsilon$ subunit $(\gamma$-AChR) may rescue the phenotype $(26,27$, 29). Patients with null or low-expressor mutations in other AChR subunits may not survive for lack of a substituting subunit. Indeed, no null or low-expressor mutations of the $\beta$ subunit have been reported to date (25).
Phenotype effects of the mutations in $\beta$ subunit of $A C h R$. Because both the patient and her affected siblings harbor 2 different mutations in the $\beta$ subunit of AChR, skipping of exon 8 and $\beta 426 \mathrm{delEQE}$, and because each mutation is carried by an unaffected parent, the 2 mutations are heteroallelic and recessive. The allele lacking exon 8 essentially abolishes AChR expression; therefore, the clinical phenotype is determined by the consequences of $\beta 426$ delEQE. The pathogenicity of $\beta 426 \mathrm{delEQE}$ is reflected by the extremely severe clinical symptoms and by a reduction of the number of AChRs per EP to approximately 7\% of normal. The sparse $\gamma$ AChR at the EPs, accounting for less than $1 \%$ of the expected normal number of receptors, are unlikely to be functionally significant. The safety margin of neuromuscular transmission is thus compromised by the decreased number of AChRs available to transmitter quanta released from the nerve terminal.

Functional and structural consequences of the $\beta 426$ delEQE mutation. Assembly of the muscle AChR is a highly orchestrated process encompassing subunit synthesis followed by cycles of folding and subunit oligomerization (30). Assembly begins with formation of an $\alpha \beta \varepsilon$ trimer, followed by specific folding steps, oligomerization of the $\delta$ subunit, additional folding, and then oligomerization of the second $\alpha$ subunit. We find the $\beta 426$ delEQE subunit reduces formation of $\alpha_{2} \beta \delta_{2}$ pentamers, but not formation of $\alpha_{2} \beta \varepsilon_{2}$ pentamers, indicating disruption of a process specific to the $\beta$ and $\delta$ subunits. Because these subunits initially associate with the $\alpha$ subunit, the $\beta-\delta$ subunit interaction we observe may depend on the presence of the $\alpha$ subunit. Therefore, addition of a $\delta$ subunit to either $\alpha \beta \varepsilon$ or $\alpha \beta \delta$ trimers is much less efficient in the presence of the $\beta 426$ delEQE subunit. Because AChR assembly is regulated to assure that only properly assembled subunits accumulate, improperly assembled subunit complexes are rapidly degraded (31). Therefore, the unassembled $\alpha \beta \varepsilon$ complex and the free $\delta$ subunit are likely rapidly degraded, reducing cell-surface expression.

The $\beta 426$ delEQE subunit likely reduces receptor expression by disrupting a secondary structural element. Deletion of 2, 3, 4, or 6 residues in this region of the $\beta$
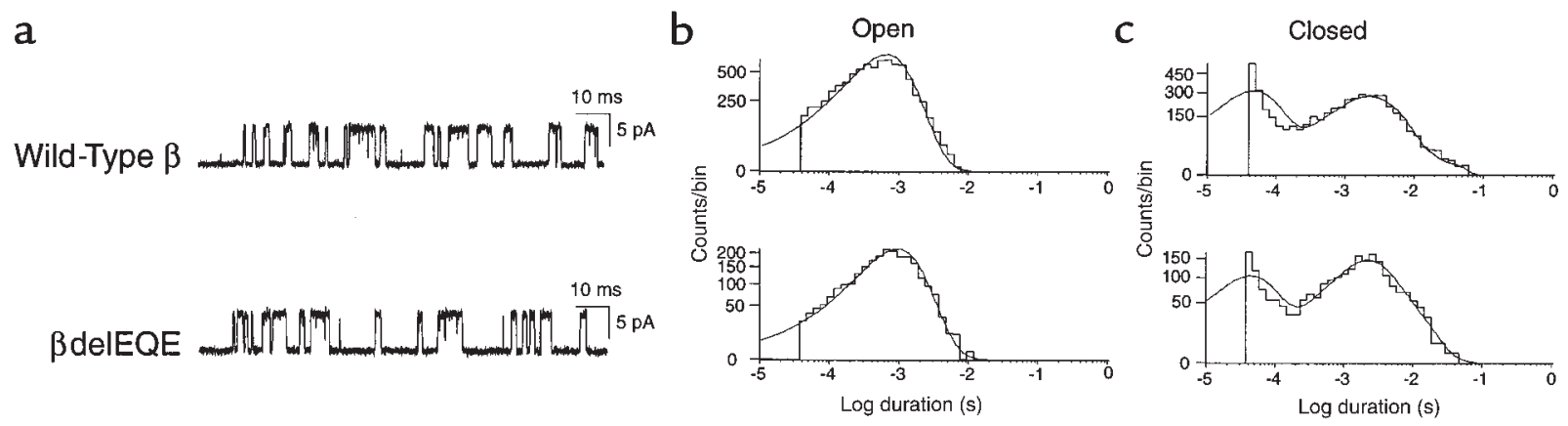

Figure 8

Single-channel currents elicited by $10 \mu \mathrm{M}$ ACh from AChRs containing wild-type or mutant $\beta$ subunits and complimentary $\alpha, \delta$, and $\varepsilon$ subunits. Currents are shown filtered at $8 \mathrm{kHz}$ with openings as upward deflections (a). Corresponding open- and closed-duration histograms (b and $\mathbf{c})$ are shown fitted by the sum of exponentials with the following parameters. Wild-type: open durations, $\tau_{\circ}=0.65 \mathrm{~ms}$, number of events $=7681$; closed durations, $\tau_{1}=0.45 \mathrm{~ms}, \mathrm{a}_{1}=0.47, \tau_{2}=2.5 \mathrm{~ms}, \mathrm{a}_{2}=0.53$. Mutant $\beta$ delEQE: open durations, $\tau_{\mathrm{o}}=0.99 \mathrm{~ms}$, number of events $=2422 ;$ closed durations, $\tau_{1}$ $=0.44 \mathrm{~ms}, \mathrm{a}_{1}=0.39, \tau_{2}=3.9 \mathrm{~ms}, \mathrm{a}_{2}=0.61$. 
subunit reduces surface expression, whereas replacing EQE with the triple mutations AAA or KQK does not (Figure 5), suggesting that $\beta 426$ delEQE disrupts a secondary structure such as an $\alpha$-helix. Additionally, both the $\mathrm{NH}_{2}$-terminal domains and the long cytoplasmic loop of the AChR are thought to contain recognition signals for AChR assembly $(2,3,32)$. In particular, deletions of the long cytoplasmic loop of the $\delta$ subunit disrupts assembly (32), but the specific residues responsible for this effect remained unidentified.

The long cytoplasmic loop of AChR subunits interacts with the protein rapsyn to cluster receptors at the EP (32). The $\beta$ subunit has been chemically cross-linked to rapsyn in Torpedo membranes (33), and the association with rapsyn metabolically stabilizes the receptor by reducing its turnover at the EP (34). Our results show that the deletion mutations in the $\beta$ subunit reduce cell surface more than total cellular expression, indicating that this region of the subunit may contribute to receptor turnover as well as to subunit oligomerization. Furthermore, the $\beta 426 \mathrm{delEQE}$ subunit reduces AChRs at the patient EP to approximately $7 \%$ of controls, but reduces AChRs in 293 HEK cells to only $30 \%$ of controls. The discrepancy between receptors at the patient EPs and 293 HEK cells likely resides in differences between posttranslational events occurring in 293 HEK cells and in the human muscle fiber. Such events could include accelerated destruction of intracellular receptor complexes or defective stabilization of the mutant receptors by rapsyn. Thus, the altered secondary structure in the long cytoplasmic loop of $\beta 293$ delEQE may compromise AChR expression at the EP, not only by preventing efficient association with the $\delta$ subunit, but also by adversely affecting interactions with junctional proteins that stabilize the receptor.

\section{Acknowledgments}

This work was supported by grants from the National Institutes of Health to A.G. Engel (NS 6277) and to S.M. Sine (NS 31744) and a Muscular Dystrophy Association Research grant to A.G. Engel.

1. Green, W.N. 1999. Ion channel assembly: creating structures that function. J. Gen. Physiol. 113:163-169.

2. Yu, X.-M., and Hall, Z.W. 1991. Extracellular domains mediating $\varepsilon$ subunit interactions of muscle acetylcholine receptor. Nature. 352:64-67.

3. Verrall, S., and Hall, Z.W. 1992. The N-terminal domains of the acetylcholine receptor subunits contain recognition signals for the initial steps of receptor assembly. Cell. 68:23-31.

4. Green, W.N., and Wanamaker, C.P. 1997. The role of the cystine loop in acetylcholine receptor assembly. J. Biol. Chem. 272:20945-20953.

5. Gu, Y., Camacho, P., Gardner, P., and Hall, Z.W. 1991. Identification of two amino acid residues in the epsilon subunit that promote mammalian muscle acetylcholine receptor assembly in COS cells. Neuron. 6:879-887.

6. Kreienkamp, H.-J., Sine, S.M., Maeda, R.K., and Taylor, P. 1994. Glycosylation sites selectively interfere with $\alpha$-toxin binding to the nicotinic acetylcholine receptor. J. Biol. Chem. 269:8108-8114.

7. Garcia-Guzman, M., Sala, F., Sala, S., Campos-Caro, A., and Criado, M. 1994. Role of two acetylcholine receptor subunit domains in homomer formation and intersubunit recognition, as revealed by $\alpha 3$ and $\alpha 7$ chimeras. Biochemistry. 33:15198-15203.

8. Fambrough, D.M., Engel, A.G., and Rosenberry, T.L. 1982. Acetylcholinesterase of human erythrocytes and neuromuscular junctions: homologies revealed by monoclonal antibodies. Proc. Natl. Acad. Sci. USA. 79:1078-1082.

9. Engel, A.G. 1994. The muscle biopsy. In Myology. 2nd edition. A.G. Engel and C. Franzini-Armstrong, editors. McGraw-Hill. New York, NY. 822-831.

10. Engel, A.G. 1994. Quantitative morphological studies of muscle. In Myology. 2nd edition. A.G. Engel and C. Franzini-Armstrong, editors. McGrawHill. New York, NY. 1018-1045.

11. Engel, A.G., Lindstrom, J.M., Lambert, E.H., and Lennon, V.A. 1977. Ultrastructural localization of the acetylcholine receptor in myasthenia gravis and in its experimental autoimmune model. Neurology. 27:307-315.

12. Engel, A.G., Nagel, A., Walls, T.J., Harper, C.M., and Waisburg, H.A. 1993. Congenital myasthenic syndromes. I. Deficiency and short open-time of the acetylcholine receptor. Muscle Nerve. 16:1284-1292.

13. Uchitel, O., et al. 1993. Congenital myasthenic syndromes. II. A syndrome attributed to abnormal interaction of acetylcholine with its receptor. Muscle Nerve. 16:1293-1301.

14. Milone, M., et al. 1997. Slow-channel syndrome caused by enhanced activation, desensitization, and agonist binding affinity due to mutation in the M2 domain of the acetylcholine receptor alpha subunit. J. Neurosci. 17:5651-5665.

15. Ohno, K., et al. 1995. Congenital myasthenic syndrome caused by prolonged acetylcholine receptor channel openings due to a mutation in the M2 domain of the $\varepsilon$ subunit. Proc. Natl. Acad. Sci. USA. 92:758-762.

16. Ohno, K., et al. 1996. Congenital myasthenic syndrome caused by decreased agonist binding affinity due to a mutation in the acetylcholine receptor $\varepsilon$ subunit. Neuron. 17:157-170.

17. Ohno, K., et al. 1998. Myasthenic syndromes in Turkish kinships due to mutations in the acetylcholine receptor. Ann. Neurol. 44:234-241.

18. Bouzat, C., Bren, N., and Sine, S.M. 1994. Structural basis of different gating kinetics of fetal and adult acetylcholine receptors. Neuron. 13:1395-1402.

19. Sigworth, F.J., and Sine, S.M. 1987. Data transformation for improved display and fitting of single-channel dwell time histograms. Biophys. J. 52:1047-1054

20. Milone, M., Hutchinson, D.O., and Engel, A.G. 1994. Patch-clamp analysis of the properties of acetylcholine receptor channels at the normal human endplate. Muscle Nerve. 17:1364-1369.

21. Mishina, M., et al. 1986. Molecular distinction between fetal and adult forms of muscle acetylcholine receptor. Nature. 321:406-411.

22. Sine, S.M., and Claudio, T. 1991. Gamma and delta subunits regulate the affinity and the cooperativity of ligand binding to the acetylcholine receptor. J. Biol. Chem. 266:19369-19377.

23. Finer-Moore, J., and Stroud, R.M. 1984. Amphipathic analysis and possible formation of the ion channel in an acetylcholine receptor. Proc. Natl. Acad. Sci. USA. 81:155-159.

24. Engel, A.G. 1994. Myasthenic syndromes. In Myology. 2nd edition. A.G. Engel and C. Franzini-Armstrong, editors. McGraw-Hill. New York, NY. $1798-1835$.

25. Engel, A.G., Ohno, K., and Sine, S.M. 1999. Congenital myasthenic syndromes: recent advances. Arch. Neurol. 56:163-167.

26. Ohno, K., et al. 1997. Congenital myasthenic syndromes due to heteroallelic nonsense/missense mutations in the acetylcholine receptor $\varepsilon$ subunit gene: identification and functional characterization of six new mutations. Hum. Mol. Genet. 6:753-766.

27. Engel, A.G., Ohno, K., Bouzat, C., Sine, S.M., and Griggs, R.G. 1996. Endplate acetylcholine receptor deficiency due to nonsense mutations in the $\varepsilon$ subunit. Ann. Neurol. 40:810-817.

28. Middleton, L., et al. 1999. Congenital myasthenic syndromes linked to chromosome $17 \mathrm{p}$ are caused by defects in acetylcholine receptor $\varepsilon$ subunit gene. Neurology. 53:1076-1082.

29. Milone, M., et al. 1998. Mode switching kinetics produced by a naturally occurring mutation in the cytoplasmic loop of the human acetylcholine receptor $\varepsilon$ subunit. Neuron. 20:575-588.

30. Green, W.N., and Wanamaker, C.P. 1998. Formation of the nicotinic acetylcholine receptor binding sites. J. Neurosci. 18:5555-5564.

31. Blount, P., and Merlie, J.P. 1990. Mutational analysis of muscle nicotinic acetylcholine receptor subunit assembly. J. Cell. Biol. 111:2613-2622.

32. Yu, X.-M., and Hall, Z.W. 1994. The role of the cytoplasmic domains of individual subunits of the acetylcholine receptor in $43 \mathrm{kDa}$ protein-induced clustering in COS cells. J. Neurosci. 14:785-795.

33. Burden, S.J., De Palma, R.L., and Gottesman, G.S. 1983. Crosslinking of proteins in acetylcholine receptor-rich membranes: association between the $\beta$ subunit and the $43 \mathrm{kD}$ subsynaptic protein. Cell. 35:687-692.

34. Wang, Z.-Z., Mathias, A., Gautam, M., and Hall, Z.W. 1999. Metabolic stabilization of muscle nicotinic acetylcholine receptor by rapsyn. J. Neurosci. 19:1998-2007 